\section{Alcohol consumption increases periodontitis risk}

Pitiphat W, Merchant AT et al. J Dent Res 2003; 82: 509-513

Alcohol use appeared to relate to later periodontitis in subjects initially reported without it.

In a longitudinal study of 51,529 male health professionals (60\% dentists) from 1986, alcohol intake was recorded in 1986, 1990 and 1994. Subjects with reported periodontitis, myocardial infarction or inadequate data were excluded from the present study, leaving 39,461. Presence of periodontitis was recorded, according to recalled history of diagnosed disease with bone loss, every 2 years to 1998; the validity of this procedure was assessed as $70-80 \%$ in a radiographed subsample.

The relative risk for periodontitis reported in 2125 subjects after 1986 was significantly greater for all levels of estimated alcohol intake when compared with 373 teetotallers. When controlled for age, smoking, diabetes and other potential confounding factors, all relative risks (means: 1.18-1.27) just reached significance, but there was no dose relationship.

Oral hygiene was not assessed objectively in this study, although a previous paper recorded self-reported oral hygiene practices in the population. It is therefore unclear whether the reported association between alcohol and periodontitis was due to behavioural or systemic factors. Assessment of alcohol intake is also very difficult with the method reported, which is not the familiar UK 'unit' system.

doi:10.1038/sj.bdj.4810594

\section{ORAL PHYSIOLOGY}

\section{Investigation of the factors related to the formation of the buccal mucosa ridging}

Takagi I, Sakurai K J Oral Rehabil2003; 30: 565-572

Clenching the teeth was not associated with mucosal ridging, but swallowing was related to cheek pressure on teeth.

Some clinicians have considered that buccal mucosa ridging adjacent to the occlusal contact of arches occurs as a consequence of tooth clenching habits. In this study, 3 groups of 7 subjects were identified with no ridging (C), ridging limited to molars (M), and ridging related to molars and premolars (MP). Mean age was 25 yrs, and all subjects had normal occlusion and dentitions.

Pressure on the middle of the maxillary first premolar and distal of the second molar was measured during a variety of clenching and oral cheek musculature tasks, including swallowing, For swallowing but not for any other activity, there was a significant difference between groups at both pressure loci, with mean scores greatest for MP and least for C (premolar: MP - $11500 \mathrm{~Pa}, \mathrm{M}-9100$ Pa, C - 600 Pa; molar: MP - 8900 Pa, M - 7400 Pa, C - 700 Pa).

ABSTRACTS

PROSTHODONTICS; IMPLANT DENTISTRY

\section{Oral and general health-related quality of life with conventional and implant dentures}

Heydecke G, Locker D etal. Community Dent Oral Epidemiol 2003; 31: 161-168

Implant-supported overdentures improved quality of life.

A comparison was made between 2 groups of edentulous Canadian patients 6 months after prosthodontic treatment. Quality of life in 25 patients with complete upper and lower conventional dentures was compared with that in 30 patients who had similar treatment but with the lower denture supported by 2 ITI implants. Groups were demographically similar, each with mean age $69 \mathrm{yrs}$, and subjects had been edentulous for 5 yrs.

Before treatment and during follow-up, specific questionnaires were used to assess general and denture-related quality of life. At baseline, groups did not differ in these respects, nor in mandibular ridge dimensions and quality of mandibular mucosa. After 6 months, oral quality of life had improved significantly more in the implant group than the conventional group, specifically with regard to function and discomfort. In overall quality of life, the implant group improved significantly in some respects but without differences between groups.

doi:10.1038/sj.bdj.4810596

\section{PERIODONTOLOGY; OBESITY}

\section{Obesity and periodontal disease in young,} middle-aged and older adults

Al-Zahrani MS, Bissada NF et al. J Periodontol 2003; 74: 610-615

These conditions were associated in younger adults.

Following reports of an association between obesity and periodontal disease, these researchers investigated the matter in a large US database on health and nutrition (NHANES III). Periodontal data for 13,665 subjects were examined in relation to body mass index and waist circumference. Periodontal disease was defined as the presence of 1 or more sites with both attachment loss $>2 \mathrm{~mm}$ and probing depth $>3 \mathrm{~mm}$.

In a multivariate statistical model including social factors, smoking and diabetes, there were significant interactions with age, so data were stratified for younger (18-34 yrs old), middleaged (35-59) and older (60-90) subjects. In the younger subjects only, there was a significant association; subjects who were underweight had a significantly lower prevalence of periodontal disease (adjusted $\mathrm{OR}=0.21$ ) than those who were normal or overweight, and those who were obese had a significantly higher prevalence (1.76). Unfortunately, plaque data were not recorded. The authors discuss possible mechanisms to account for their results.

doi:10.1038/sj.bdj.4810597 